\title{
Clarithromycin-induced Coronary Vasospasms Caused Acute Coronary Syndrome in a 19-year-old Male Patient
}

\author{
Masahiro Ishikura, Akihiro Endo, Takahiro Sakamoto, Junya Tanabe, Koichi Okazaki, \\ Takeshi Ouchi, Nobuhide Watanabe and Kazuaki Tanabe
}

\begin{abstract}
:
A 19-year-old-man was admitted to our hospital with intermittent chest pain. The day before admission, he had been diagnosed with enteritis and prescribed clarithromycin. He had experienced severe chest pain three times after taking clarithromycin; thus, acute coronary syndrome (ACS) was suspected. Emergent coronary angiography showed normal coronary arteries; however, the result of a subsequent acetylcholine provocation test was positive. We diagnosed him to have ACS caused by coronary vasospasms and suspected clarithromycin-induced Kounis syndrome. Although more common in older patients, Kounis syndrome must be suspected and a thorough medication history should be taken whenever a patient complains of chest pain.
\end{abstract}

Key words: coronary vasospasms, adolescent, clarithromycin, Kounis syndrome

(Intern Med 60: 281-285, 2021)

(DOI: 10.2169/internalmedicine.5548-20)

\section{Introduction}

Vasospastic angina (VSA) usually develops after middle age (1-3), however, it is extremely rare in adolescents $(4,5)$. Kounis syndrome is commonly known as an allergic angina or an allergic myocardial infarction caused by coronary vasospasms (6), which may also occur in adolescence (7). It is often caused by antibiotics, including clarithromycin $(7,8)$. Recently, several studies have reported an increased risk of cardiovascular mortality in patients taking clarithromycin (9-11). Based on these reports, the U. S. Food and Drug Administration has called attention to the risks involved when prescribing clarithromycin to patients with heart disease (12).

In this case report, we present the case of a 19-year-old male patient suspected to have Kounis syndrome caused by clarithromycin in the development of acute coronary syndrome (ACS).

\section{Case Report}

A 19-year-old-man was admitted to our hospital with intermittent chest pain. He was previously healthy with no coronary risk factors for hyperlipidemia, diabetes mellitus, Kawasaki disease, family history of heart disease, or smoking and passive smoking history. On the day before admission, he had consulted a nearby hospital for fever and diarrhea. He was diagnosed with enteritis, and as a result, he was prescribed oral clarithromycin. At 4 AM, approximately 6 hours after taking clarithromycin, he felt severe chest pains that he had previously not experienced before. However, the symptoms disappeared spontaneously within 1 hour. He took clarithromycin again after breakfast, and similar chest pains recurred at 12:00 PM and continued for 1 hour. Once again, the patient took clarithromycin after dinner, and several hours later, he experienced chest pains; therefore, he visited the nearby hospital again. ACS was suspected, so he was transferred to our hospital. The chest pain had already disappeared when he arrived at our hospital. On physical examination, his level of consciousness was normal, blood pressure was $138 / 81 \mathrm{mmHg}$, pulse was 64 beats per minute, temperature was $37.6^{\circ} \mathrm{C}$, and oxygen saturation was $100 \%$. He had no cardiac murmur or wheezing on auscultation. No eruptions, decrease in blood pressure or asthmatic bronchial reaction were observed. There were no abnormal findings in all other parameters of the physical examination. An electrocardiogram revealed terminal $\mathrm{T}$ wave 
(A)

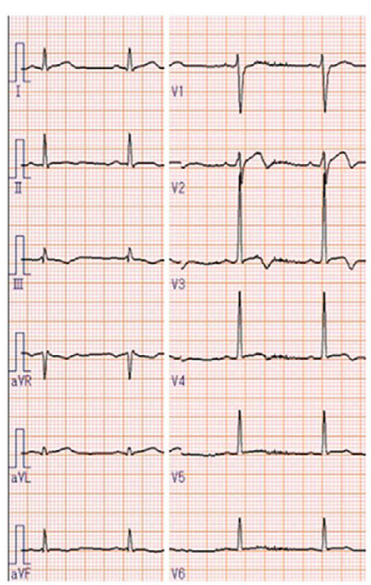

(C)

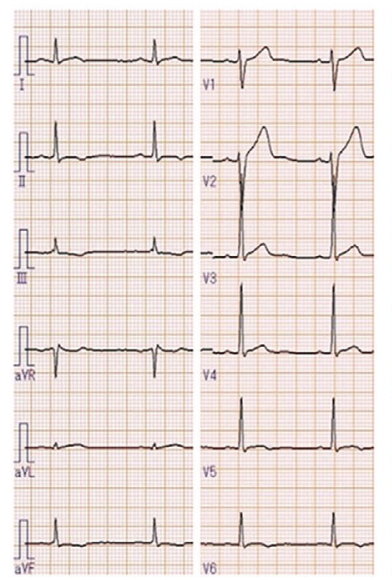

(B)

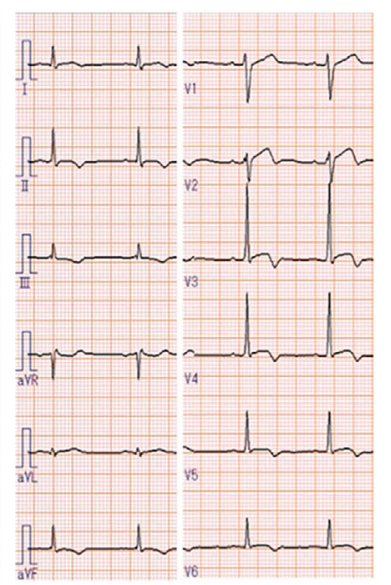

(D)

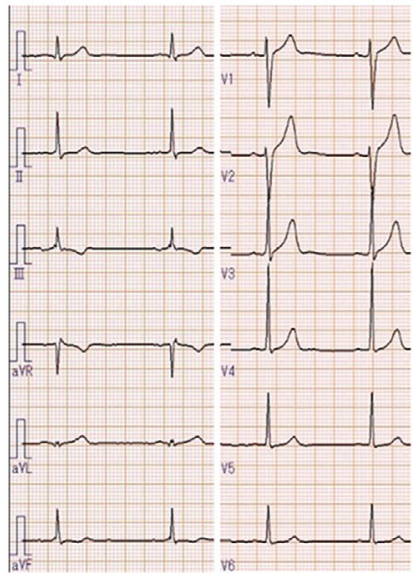

Figure 1. An electrocardiogram. Terminal T wave inversion in V1 to V4 was found on admission (A). However, on the following day, negative $\mathrm{T}$ waves without a prolonged QT interval (QTc $410 \mathrm{~ms}$ ) had developed in the inferior leads (B). The abnormal findings gradually improved before discharge (on day 7) (C), and only negative T wave in III lead remained at the time of admission for the second acetylcholine provocation test (D).

inversion in V1 to V4 (Fig. 1A). Laboratory tests showed elevated troponin I (10.93 ng/mL), creatine kinase (CK; 802 $\mathrm{U} / \mathrm{L})$ and CK-MB (54.8 ng/mL) levels; however, WBC was within normal limits $(8,140 / \mu \mathrm{L})$. We suspected ACS; however, emergent coronary angiography showed normal coronary arteries (Fig. 2A). Subsequently, we performed an acetylcholine provocation test, wherein the administration of 50 $\mu \mathrm{g}$ acetylcholine into the right coronary artery induced chest pains similar to those that he had previously experienced. On an electrocardiogram, transient ST segment depression and negative $\mathrm{T}$ wave appeared in the inferior wall leads (Fig. 3). In addition, coronary vasospasms, which resulted in 99\% stenosis in the ostium of the posterior descending artery, were observed during the test (Fig. 2B). Left ventriculography showed akinesis in the narrow area of the inferior wall (Fig. 4), and the patient was subsequently diagnosed

with vasospastic angina. Combination therapy with nitric acid and calcium-channel blocker was commenced, and clarithromycin was stopped. An electrocardiogram conducted the following day revealed negative $\mathrm{T}$ waves without any QT interval prolongation in the inferior leads. These electrocardiographic abnormal findings gradually improved before discharge (Fig. 1C), however, the improvement in inferior wall motion on echocardiogram was limited. The CK levels were the highest on admission, which was 18 hours after first attack, and they decreased thereafter, normalizing 1.5 days later. The chest symptoms did not recur during hospitalization, and he was discharged 9 days later without any complications. He had never experienced any chest pain previously; however, severe chest pain due to coronary vasospasm occurred three times after he took clarithromycin. An association between taking clarithromycin and coronary vasospasms was suspected. The value of non-specific IgE on day 2 was very high $(375 \mathrm{IU} / \mathrm{mL}, \mathrm{N}$ : <87). Consequently, we performed a drug-induced lymphocyte stimulation test (DLST) for clarithromycin, and the results were strongly positive (stimulation index 449\%; >180 was judged positive). As a result, we thought that the coronary spasm may have been induced by an allergic reaction to clarithromycin.

After 3 months, coronary angiography with an acetylcholine provocation test was performed again. At this admission, the electrocardiographic findings were improved and only a negative $\mathrm{T}$ wave in the III lead remained (Fig. 1D), and the wall motion in the inferior wall improved to hypokinesis on an echocardiogram. He was admitted 2 days before the examination, and taking calcium-channel blockers and nitrate were discontinued more than 48 hours before the acetylcholine provocation test. This time, the administration of $50 \mu \mathrm{g}$ acetylcholine did not induce significant vasospasms (Fig. 5), there were no chest pains, and electrocardiogram changes were also not observed. Therefore, the acetylcholine provocation test was judged to be negative. Accordingly, the patient was banned from taking clarithromycin and continued taking prophylactic calcium-channel blockers. Thereafter, the recurrent chest pain never occurred.

\section{Discussion}

VSA is predominantly observed in patients over 40 years of age. The pathophysiology is thought to be vascular smooth muscle hyper-reactivity and endothelial dysfunction (1-3). Therefore, the incidence of VSA in young patients, especially in adolescents, is very rare $(4,5)$. We encountered a rare case of a 19-year-old patient with VSA. He had never been aware of chest pain prior to this episode. However, severe chest pain occurred repeatedly several hours after he took clarithromycin, which caused ACS. We suspected the chest pain "storm" was related to clarithromycin. Clarithromycin is an antibiotic that is frequently prescribed in clinical practice in Japan. However, recently, the U. S. Food and Drug Administration warned about a potential increased risk of heart problems with clarithromycin 


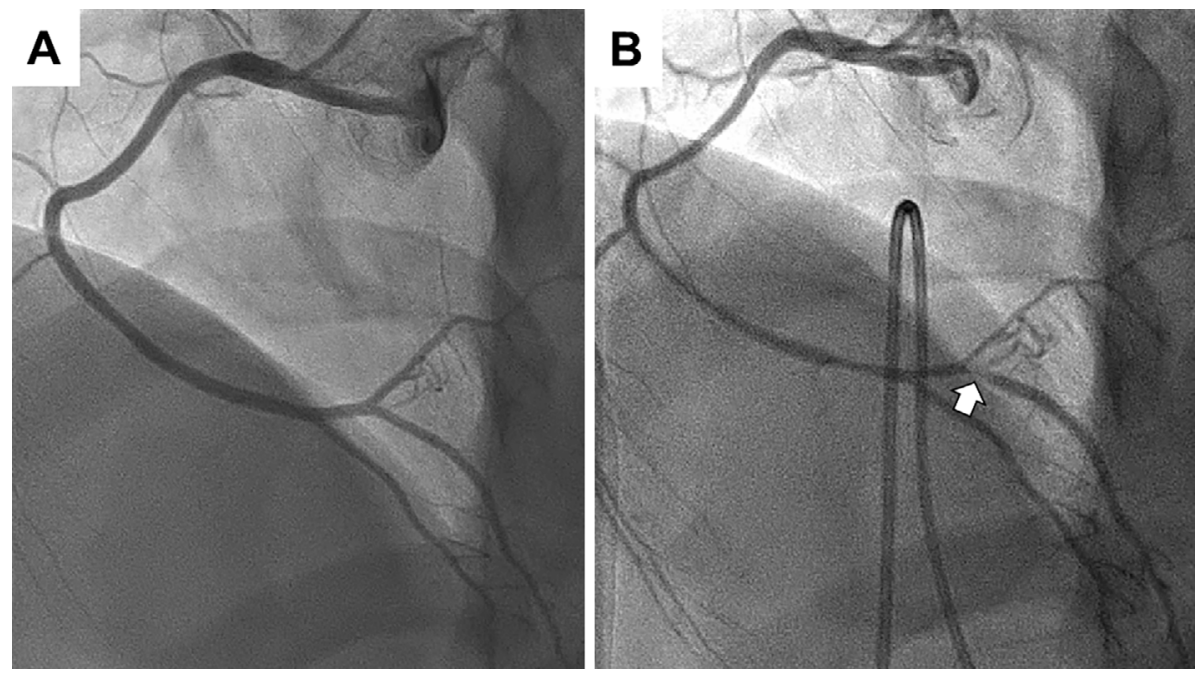

Figure 2. Coronary angiograms before and after the administration of acetylcholine on emergency admission. Although there was no significant stenosis in the control (A), the administration of $50 \mu \mathrm{g}$ acetylcholine triggered coronary vasospasms, inducing $99 \%$ stenosis in the ostium of the posterior descending artery (white arrow), with chest pains (B).

(A)

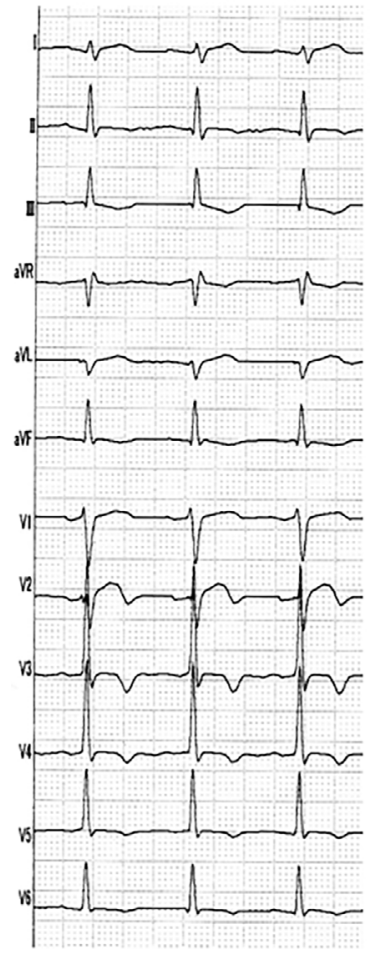

(B)

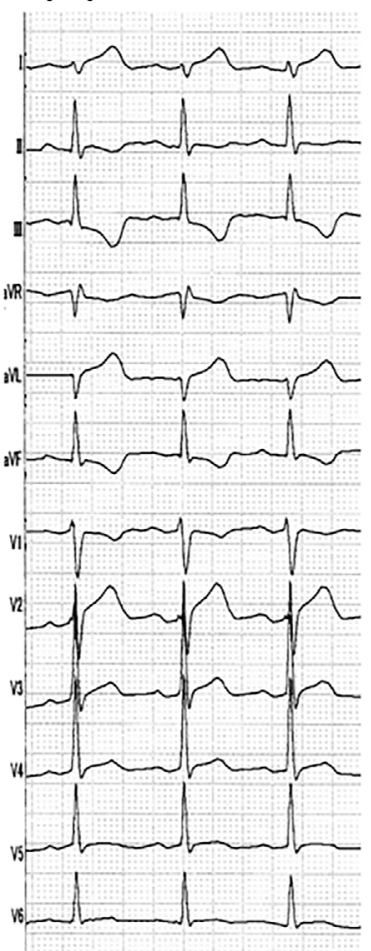

Figure 3. The electrocardiograms before (A) and during (B) the administration of acetylcholine. ST segment depression and negative $T$ wave appeared in the inferior wall leads.

use (12). The report advised prescribers to consider using other antibiotics in patients with heart disease. The CLARICOR trial, a prospective, randomized, multicenter trial, showed that short-term clarithromycin prescribed for patients with stable coronary disease caused significantly higher cardiovascular mortality during a 3-year follow-up period (9). A long term follow-up of the CLARICOR trial also showed

that clarithromycin increased cardiovascular mortality by over 10 years in patients with coronary heart disease who were not on statins (10). Similarly, in a large populationbased cohort study including 326,781 patients, clarithromycin increased the risk of myocardial infarction within 14 days after start of treatment compared to amoxicillin (11). The exact mechanism of how clarithromycin induces more adverse cardiovascular events remains unclear. A prolonged QT interval, caused by the potassium channel blocking effect of clarithromycin, was considered to be a possible cause (13). Kounis syndrome induced by clarithromycin might be a reason for the incidence of myocardial infarctions and increase in cardiovascular mortality.

Angina and myocardial infarctions caused by allergic coronary vasospasms were first reported in 1991, and it is known as Kounis syndrome. Hypersensitive coronary disorders induced by various conditions, drugs, foods, and environmental exposures are suspected to cause Kounis syndrome (6-8). Of the drugs that often cause this syndrome, antibiotics were the most common triggers (7). In the PubMed database, our search uncovered 1 case report of 36year-old patient with Kounis syndrome caused by clarithromycin (14). There are no standard criteria to diagnose Kounis syndrome, so it is based on clinical symptoms, laboratory, electrocardiograms, echocardiograms, or angiographic evidence. The main cause of Kounis syndrome is thought to be a type I allergic reaction (7). DLST is a method to determine whether the patient has a hypersensitivity reaction of T-cell. Because there are such T-cell responses in all types of allergic reactions, DLST has been found to be frequently positive not only in delayed hypersensitivity reactions but also in anaphylaxis (>50\%) (15). We think that the high value of IgE and positive result of DLST for clarithromycin in our patient might suggest that he had some kind of allergy to clarithromycin, which thereby triggered coronary 


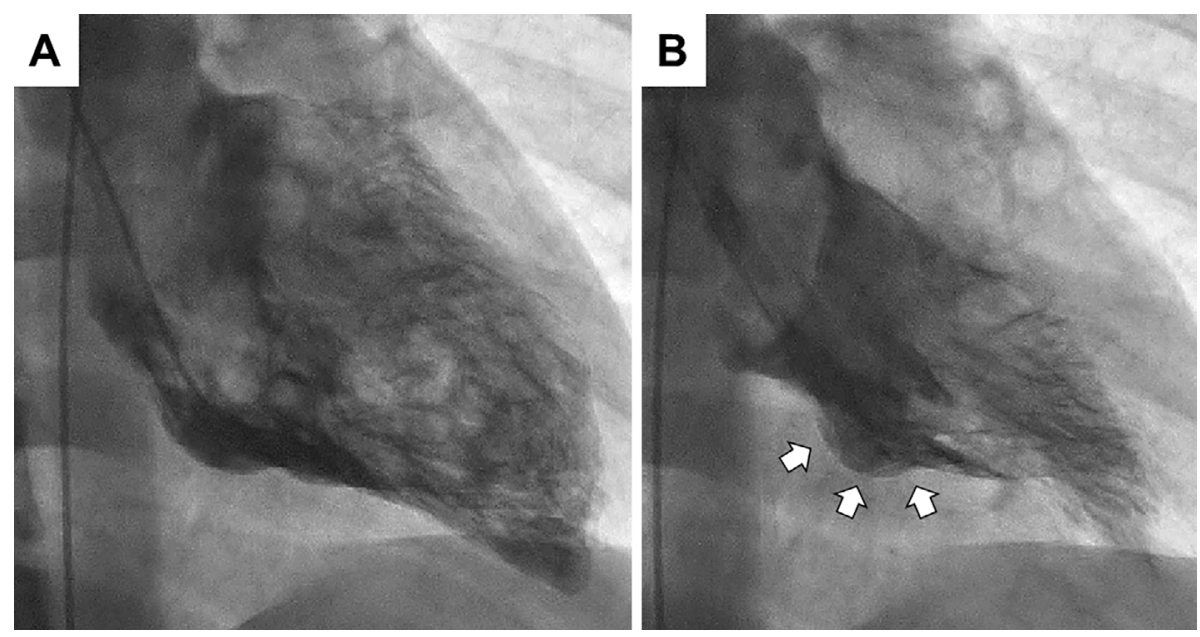

Figure 4. Left ventriculography on emergency admission. Akinesis in the narrow area of the inferior wall (white arrows) was noted (A: diastolic phase, B: systolic phase).
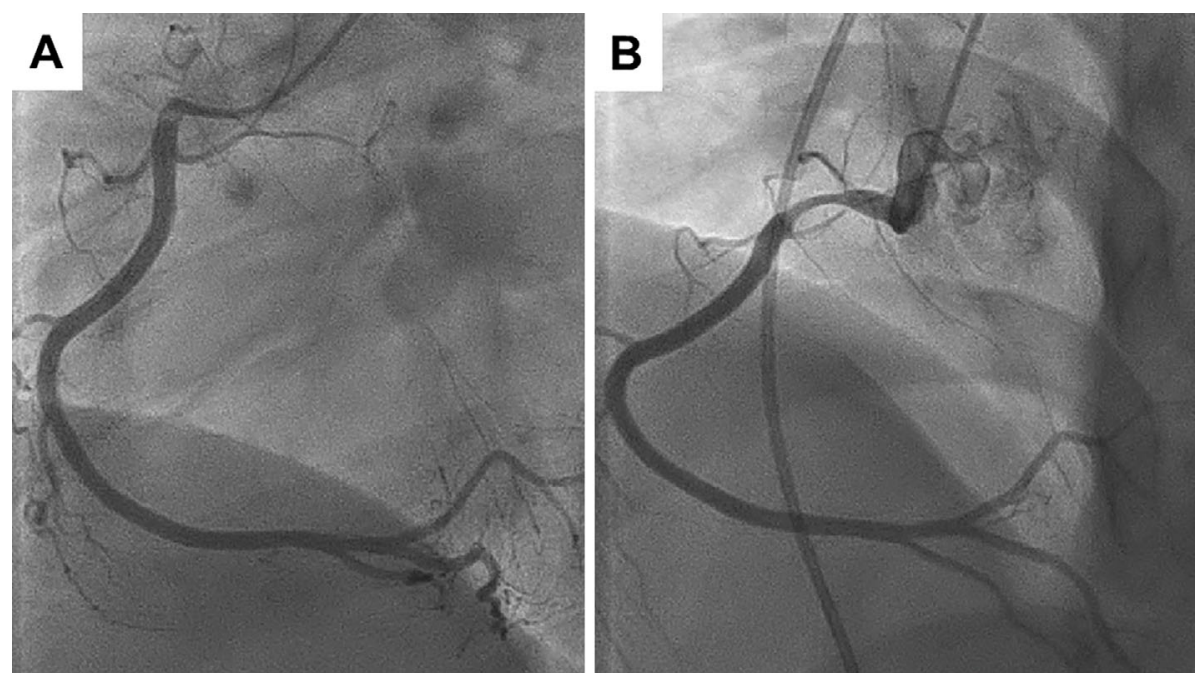

Figure 5. Coronary angiograms before (A) and after (B) the administration of acetylcholine after 3 months. Significant vasospasms and chest pain were not induced by acetylcholine. The stenotic lesion in the proximal right coronary artery was thought to be a catheter-induced spasm.

spasms, resulting in ACS.

Takotsubo cardiomyopathy may be considered to be an important differential diagnosis in such cases (16). However, it is often triggered by emotional stress (17), and there was no episode of stress in our patient. Furthermore, the characteristic findings of takotsubo cardiomyopathy, such as apical ballooning of left ventricular motion and QT interval prolongation with giant negative $\mathrm{T}$ waves on electrocardiogram, were not observed (18). Myocarditis caused by bacteria or virus which were related to enteritis is another possibility when making a differential diagnosis. However, in the typical case of myocarditis, the systolic dysfunction is generally diffuse (19). We believe that segmental left ventricular wall akinesis and rapid normalization of cardiac enzymes after admission were more consistent with ACS caused by coronary vasospasms than takotsubo cardiomyopathy and myocarditis. Moreover, there might be a possibility that enteritis had caused coronary vasospasm. However, there was no such case report, as far as we could search. Because severe chest pain occurred repeatedly every time he took clarithromycin, we believed that clarithromycin-induced Kounis syndrome was the most likely cause of this episode. Continuation of taking calcium-channel blockers may not be essential. However, in consideration of the possibility of Kounis syndrome recurrence by some other allergen, we continued calcium-channel blockers with his own request.

\section{Conclusions}

We herein described the case of a 19-year-old male patient diagnosed with ACS caused by coronary vasospasms, where clarithromycin was suspected to be a contributing factor. We propose that even in case of an adolescent complaining of chest pain, it is necessary to consider Kounis syndrome and to take a detailed medication history. Moreover, when prescribing clarithromycin to patients with cardiovascular diseases, it is important to explain that if any chest 
discomfort occurs, they should visit the hospital immediately.

The authors state that they have no Conflict of Interest (COI).

\section{References}

1. JCS Joint Working Group. Guidelines for diagnosis and treatment of patients with vasospastic angina (Coronary Spastic Angina) (JCS 2013). Circ J 78: 2779-2801, 2014.

2. Yasue H. Pathophysiology and treatment of coronary arterial spasm. Chest 78: 216-223, 1980.

3. Beltrame JF, Crea F, Kaskiet JC, et al. The who, what, why, when, how and where of vasospastic angina. Circ J 80: 289-298, 2016.

4. Sueda S. Young vasospastic angina patients less than 20 years old. Circ J 83: 1925-1928, 2019.

5. Kobayashi K, Kouzuma R, Tsutsui M, Okazaki M, Tasaki H, Nakashima Y. Vasospastic angina in a 16-year-old female. Circ J 67: 467-469, 2003.

6. Kounis NG, Zavras GM. Histamine-induced coronary artery spasm: the concept of allergic angina. Br J Clin Pract 45: 121$128,1991$.

7. Abdelghany M, Subedi R, Shah S, Kozman H. Kounis syndrome: a review article on epidemiology, diagnostic findings, management and complications of allergic acute coronary syndrome. Int J Cardiol 232: 1-4, 2017.

8. Kounis NG. Kounis syndrome: an update on epidemiology, pathogenesis, diagnosis and therapeutic management. Clin Chem Lab Med 54: 1545-1559, 2016.

9. Jespersen CM, Als-Nielsen B, Damgaard M, et al. Randomized placebo controlled multicenter trial to assess short term clarithromycin for patients with stable coronary heart disease: CLARICOR trial. BMJ 332: 22-27, 2006.

10. Winkel P, Hilden J, Hansen JF, et al. Clarithromycin for stable coronary heart disease increases all-cause and cardiovascular mortality and cerebrovascular morbidity over 10 years in the CLARICOR randomised, blinded clinical trial. Int J Cardiol 182: 459465, 2015.

11. Wong AYS, Root A, Douglas IJ, et al. Cardiovascular outcomes associated with use of clarithromycin: population-based study. BMJ 352: h6926, 2016.

12. FDA Drug Safety Communication. FDA review finds additional data supports the potential for increased long-term risks with antibiotic clarithromycin (Biaxin) in patients with heart disease [Internet]. Available from: https://www.fda.gov/media/111300/download

13. Martin D, Bursill J, Qui MR, Breit SN, Campbell T. Alternative hypothesis for efficacy of macrolides in acute coronary syndromes. Lancet 351: 1858-9, 1998.

14. Bilgin M, Akyel A, Doğan M, Sunman H, Yeter E. Acute coronary syndrome secondary to clarithromycin: the first case and review of the literature. Turk Kardiyol Dern Ars 42: 461-463, 2014.

15. Pichler WJ, Tilch J. The lymphocyte transformation test in the diagnosis of drug hypersensitivity. Allergy 59: 809-820, 2004.

16. Vultaggio A, Matucci A, Pace SD, et al. Tako-Tsubo-like syndrome during anaphylactic reaction. Eur J Heart Fail 9: 209-211, 2007.

17. Abhishek Maiti A, Dhoble A. Takotsubo cardiomyopathy. N Engl J Med 377: e24, 2017.

18. Templin C, Ghadri JR, Diekmann J, et al. Clinical features and outcomes of takotsubo (stress) cardiomyopathy. N Engl J Med 373: 929-38, 2015.

19. Guidelines for Diagnosis and Treatment of Myocarditis (JCS 2009) [Internet]. Available from: https://www.j-circ.or.jp/cms/wp-c ontent/uploads/2020/02/JCS2009_izumi_h.pdf (in Japanese)

The Internal Medicine is an Open Access journal distributed under the Creative Commons Attribution-NonCommercial-NoDerivatives 4.0 International License. To view the details of this license, please visit (https://creativecommons.org/licenses/ by-nc-nd/4.0/).

(C) 2021 The Japanese Society of Internal Medicine Intern Med 60: 281-285, 2021 